\title{
Registros de sanguijuelas de Costa Rica y clave para la identificación de las especies con redescripción de Cylicobdella costaricae
}

\section{Records of leeches from Costa Rica and taxonomic key for species identification with a redescription of Cylicobdella costaricae}

\author{
Alejandro Oceguera-Figueroa ${ }^{1,2 \otimes}$ y Bernald Pacheco-Chaves ${ }^{3}$ \\ ${ }^{1}$ Laboratorio de Helmintología, Instituto de Biología, Universidad Nacional Autónoma de México. Tercer circuito s/n, Ciudad Universitaria, Copilco, \\ Coyoacán. Apartado postal 70-153, 04510 México, D. F., México. \\ ${ }^{2}$ Laboratorio de Genética Evolutiva, Instituto Cavanilles de Biodiversidad y Biología Evolutiva, Universidad de Valencia, Valencia, España. \\ ${ }^{3}$ Escuela de Biología, Universidad de Costa Rica, 11501-2060, San José, Costa Rica. \\ \aoceguera@amnh.org
}

\begin{abstract}
Resumen. En contraste con los enormes esfuerzos realizados por caracterizar la fauna y flora de Costa Rica, con excepción de algunos registros aislados, muy poco se sabe sobre las sanguijuelas (Annelida: Clitellata) en este país. En este trabajo, presentamos la redescripción de una especie terrestre muy poco conocida: Cylicobdella costaricae, caracterizando su morfología externa e interna; esta última se mantenía completamente desconocida hasta la fecha. Adicionalmente, presentamos la primera lista de especies de sanguijuelas para Costa Rica con un total de 12 taxones registrados en las 7 provincias del país. Se incluye una breve descripción de su morfología, fotografías y localidades para cada taxón. Los registros que presentamos son el resultado del estudio de los ejemplares depositados en el Museo de Zoología de la Universidad de Costa Rica así como de recolectas recientes. Finalmente, incluimos una clave taxonómica para la identificación de las especies de sanguijuelas de Costa Rica.
\end{abstract}

Palabras clave: Hirudinea, hirudíneos, Rhynchobdellida, Arhynchobdellida, Centroamérica, clave taxonómica.

\begin{abstract}
In contrast to the enormous efforts made to characterize the fauna and flora from Costa Rica, except for a few isolated records, very little is known about leeches (Annelida: Clitellata) in the country. In this paper, we present the redescription of a poorly known terrestrial leech: Cylicobdella costaricae, characterizing its external and internal morphology, the latter, completely unknown to date. In addition, we report the first list of leech species for Costa Rica, including in total 12 taxa with records from the 7 provinces of the country. We provide a brief description of the leech morphology, pictures and localities for each taxon. The information shown here is the result of the study of both, specimens deposited at the Museo de Zoología of the Universidad de Costa Rica and the new collections. Finally, we provide a taxonomic key for species identification.
\end{abstract}

Key words: Hirudinea, hirudineans, Rhynchobdellida, Arhynchobdellida, Central America, taxonomic key.

\section{Introducción}

Los hirudíneos o sanguijuelas (Annelida: Clitellata) del continente americano han sido estudiados intensivamente desde el punto de vista taxonómico por diversos autores. En América del norte por ejemplo, los trabajos de Caballero (1941; 1956; 1959), Moore (1953), Sawyer (1967), Ringuelet (1981a), Klemm (1982), Davies y Govedich (2001) y Madilly Hovingh (2007); en América del sur, Blanchard (1849), Cordero (1937) y Ringuelet (1985) contribuyeron significativamente en la caracterización de la fauna de hirudíneos del continente. A pesar de esto, algunos países se encuentran escasamente estudiados, siendo Costa Rica un buen ejemplo de ello. Recientemente, se han realizado

Recibido: 02 mayo 2011; aceptado: 30 julio 2012 diversos estudios encaminados a la caracterización de los invertebrados de agua dulce de Costa Rica (Pacheco-Chaves, 2010; Springer et al., 2010) en los cuales el estudio de las sanguijuelas no fue abordado. A la fecha, los registros sobre hirudíneos del país son escasos y se han publicado de manera aislada (i.e. Dequal, 1917; Plotnikow, 1905; Ringuelet, 1982). Plotnikow (1905) estudió la morfología de diversas especies de sanguijuelas del mundo, entre ellas, una especie de sanguijuela de Costa Rica: Cylicobdella costaricae (Plotnikow, 1905), de la que únicamente fueron descritas las características externas. Sawyer (1986) consideró a C. costaricae como miembro de la subfamilia Cylicobdellinae (Ringuelet, 1974), en la que se reconocen 3 géneros de sanguijuelas macrófagas y terrestres distribuidas exclusivamente en América del Sur: Cylicobdella (Grube, 1871), Blanchardiella (Weber, 1913) y Lumbri- 
cobdella Kennel, 1886. La separación de los 2 primeros géneros es motivo de controversia ya que el conocimiento de su morfología interna es escaso. Cylicobdellinae junto con Gastrostomobdellinae (Richardson, 1971) han sido consideradas como parte de la familia Cylicobdellidae por Sawyer (1986); sin embargo, la monofilia del grupo ha sido puesta en duda recientemente (Oceguera-Figueroa et al., 2011; Nakano et al., 2012). En el presente trabajo se presenta la redescripción morfológica de C. costaricae, tanto externa como internamente, con base en ejemplares recolectados recientemente por los autores y con ejemplares depositados en la Colección del Museo de Zoología de la Universidad de Costa Rica. Adicionalmente, se presenta la primera lista de las especies de sanguijuelas para el país con fotografías y una breve descripción taxonómica, así como una clave dicotómica para su determinación.

\section{Materiales y métodos}

Se revisaron ejemplares de hirudíneos de 12 especies recolectadas en las 7 provincias que comprenden la República de Costa Rica (Cuadro 1). Las sanguijuelas se recolectaron de acuerdo al tipo de hábitat estudiado: la búsqueda de organismos terrestres se realizó bajo piedras y troncos, los organismos acuáticos de vida libre se buscaron bajo piedras y entre las raíces de plantas acuáticas a las orillas de ríos y lagunas. Las especies parásitas fueron recolectadas directamente de sus huéspedes. Los ejemplares recolectados se mantuvieron en recipientes de plástico con agua del medio y posteriormente se anestesiaron mediante la aplicación gradual de alcohol $96^{\circ}$ hasta que los movimientos de éstas cesaron completamente. Los ejemplares se fijaron y conservaron en alcohol al $96^{\circ}$. El material de sanguijuelas depositado en el Museo de Zoología de la Universidad de Costa Rica se estudió morfológicamente y las especies se identificaron mediante claves especializadas (Ringuelet, 1985; Sawyer, 1986). Para ello, se diseccionaron bajo un microscopio binocular Nikon SMZ-U y se fotografiaron con una cámara digital SONY $\alpha-330$ adaptada al microscopio. La ilustración de la morfología interna de Cylicobdella costaricae se realizó mediante la superposición de trazos sobre una imagen utilizando el programa Adobe Illustrator (Adobe Systems, San José, California).

\section{Redescripción}

Orden Arhynchobdellida Blanchard, 1894

Familia Cylicobdellidae Sawyer, 1986

Género Cylicobdella Grube, 1871

Cylicobdella costaricae Plotnikow, 1905 (Figs. 1-3).

Se realizó con base en 7 ejemplares.
Material examinado: tres ejemplares recolectados el 27 de noviembre, 2007 en la Escuela de Biología, Universidad de Costa Rica, San José, Costa Rica por Alejandro Oceguera-Figueroa y Bernald Pacheco-Chaves. Un ejemplar recolectado en enero de 1994 en el Parque Nacional de Guanacaste, estación Pitilla, Guanacaste, Costa Rica, por C. Moraga. Tres ejemplares recolectados el 2 agosto de 1984 en las nubes de Coronado, San José, por Bernal Morera.

Morfología externa. Cuerpo firme, muscular, largo y angosto (Figs. 1, 2). Diámetro poco variable longitudinalmente, cuerpo no aplanado, circular o semicircular en corte transversal. Color gris o pardo claro, uniforme, sin franjas ni pigmentos de disposición metamérica, región ventral ligeramente más clara. Ventosas anterior y posterior muy poco desarrolladas y en ningún caso más anchas que el cuerpo. Sin papilas, sensilas u otros órganos sensoriales distinguibles externamente. Longitud del cuerpo $74.5 \mathrm{~mm}(51-100.5)$, ancho $5.49 \mathrm{~mm}(3-8)(\mathrm{n}=10)$. Clitelo no definido. Tres pares de manchas oculares, en III b2, IV b2, V b2. Gonoporo masculino en somito XII entre los anillos b2/a2, gonoporo femenino en XII sobre b5. 1+1/2 anillos entre los gonoporos. Nefridioporos no observables externamente. Somitos I y II formados por un solo anillo, somitos III-VI formados por 2 anillos, VII y VIII formados por 3 anillos, IX con 4 anillos, X-XXIV formados por 5 anillos, XXV con 4 anillos, XXVI con 3 anillos y XXVII con 2 anillos. Ano en la superficie dorsal entre XXVI y XXVII. Dos anillos entre el ano y la ventosa posterior.

Morfología interna. Boca amplia situada en el borde posterior de la ventosa oral. Faringe de VII a XIII con 3 grandes pliegues musculares sin mandíbulas. Buche de XIV a XIX. Sin ciegos gástricos. Recto corto, sin ciegos, que desemboca dorsalmente en el ano. Testisacos bien desarrollados formando dos hileras laterales a lo largo del cuerpo, un único par por somito situados en los espacios interganglionares, 10 pares en total, de XV/XVI a XXIV/ XXV. Los vasos eferentes de cada testisaco desembocan en un vaso deferente a cada lado del cuerpo, el cual alcanza su punto más anterior en XII. Los ductos eyaculatorios presentan un extenso recorrido descendente hasta XVI y a continuación presentan nuevamente un recorrido ascendente hasta alcanzar XII, a lo largo del cual su diámetro va aumentando gradualmente (epidídimos sensu Ringuelet, 1944). Finalmente, cada uno de los ductos eyaculatorios desemboca independientemente en los cuernos atriales, los cuales están bien desarrollados y presentan 2 o más plegamientos. Ovarios simples y tubulares, se extienden desde el gonoporo femenino hasta XV. No presentan vagina ni oviducto común (Fig. 3).

Otros ejemplares estudiados: 3 ejemplares UCR19-01. Los ejemplares sin datos de recolecta fueron estudiados 
Cuadro 1. Especies, localidades, recolectores y fechas de recolecta de las sanguijuelas estudiadas en este trabajo

\begin{tabular}{|c|c|c|c|c|}
\hline Taxon & Sitio & Localidad & Recolector & Fecha \\
\hline \multicolumn{5}{|l|}{ Arhynchobdellida } \\
\hline \multirow[t]{6}{*}{ Cylicobdella costaricae } & Ojo de agua, Turrialba & Cartago & J. Мoya & $26 / 12 / 2008$ \\
\hline & $\begin{array}{l}\text { Reserva Leonel Oviedo, Campus Universidad } \\
\text { de Costa Rica, San Pedro de Montes de Oca }\end{array}$ & San José & $\begin{array}{l}\text { A. Oceguera y B. } \\
\text { Pacheco }\end{array}$ & $26 / 11 / 2007$ \\
\hline & $\begin{array}{l}\text { Estación Barva, Parque Nacional Braulio } \\
\text { Carrillo }\end{array}$ & Heredia & ND & $\mathrm{ND} / 04 / 09$ \\
\hline & $\begin{array}{l}\text { Universidad de Costa Rica, San Pedro de } \\
\text { Montes de Oca }\end{array}$ & San José & J. Moya & 19/05/2009 \\
\hline & San Pedro de Montes de Oca & San José & B. Pacheco & $12 / 04 / 2011$ \\
\hline & San Pedro de Montes de Oca, 1200msnm & San José & B. Pacheco & $\mathrm{ND} / 05 / 2011$ \\
\hline \multirow[t]{17}{*}{ Barbronia weberi } & $\begin{array}{l}\text { Ayala, Escazú; camino al club campestre entre } \\
\text { San Rafael y Ayala }\end{array}$ & San José & M. Herrera & $23 / 11 / 2008$ \\
\hline & $\begin{array}{l}\text { Ipis de Goicoechea calle a Coronado. } 150 \\
\text { metros norte de la Escuela Los Ángeles. Calle } \\
\text { La Juliana. Contiguo al puente principal }\end{array}$ & San José & M. Herrera & $19 / 04 / 2009$ \\
\hline & $\begin{array}{l}\text { San Antonio de Escazú; calle a Torrotillo, sobre } \\
\text { Río Agres }\end{array}$ & San José & M. Herrera & $23 / 11 / 2008$ \\
\hline & $\begin{array}{l}\text { Dulce Nombre de Coronado de la iglesia de Los } \\
\text { Ángeles } 26 \mathrm{~m} \text { este y } 75 \text { sur. Punto ubicado al } \\
\text { final de la Calle }\end{array}$ & San José & M. Herrera & $22 / 09 / 2008$ \\
\hline & $\begin{array}{l}\text { Barva. Primer puente entre Barva y San Pedro } \\
\text { de Barva }\end{array}$ & Heredia & M. Herrera & $10 / 02 / 2009$ \\
\hline & $\begin{array}{l}\text { Concepción de Alajuelita; intercepción de } \\
\text { Concepción hacia El Tejar }\end{array}$ & San José & M. Herrera & $03 / 11 / 2008$ \\
\hline & $\begin{array}{l}\text { Puente en la Cervecería . Calle entre Río } \\
\text { Segundo de Alajuela y San Joaquín de Flores }\end{array}$ & Heredia & M. Herrera & 06/10/2009 \\
\hline & $\begin{array}{l}\text { Del Mall Internacional } 300 \text { metros hacia el } \\
\text { aeropuerto (Phillips) } 20 \text { metros aguas abajo del } \\
\text { puente peatonal }\end{array}$ & Alajuela & M. Herrera & 06/10/2009 \\
\hline & Ingenio Taboga, Canal de riego, Cañas & Guanacaste & P. Gutiérrez & $31 / 01 / 2008$ \\
\hline & Río Pará Blanco, Moravia & San José & $\begin{array}{l}\text { P. Gutiérrez y B. } \\
\text { Pacheco }\end{array}$ & $01 / 02 / 2009$ \\
\hline & $\begin{array}{l}\text { Quebrada Los Negritos, Campus UCR, San } \\
\text { Pedro de Montes de Oca }\end{array}$ & San José & $\begin{array}{l}\text { A. Oceguera y B. } \\
\text { Pacheco }\end{array}$ & $26 / 11 / 2007$ \\
\hline & Río Toyogres & Cartago & P. Gutiérrez & $\mathrm{ND} / 02 / 2009$ \\
\hline & $\begin{array}{l}\text { Santo Domingo . Calle a Tibás. Plantel Colima } \\
\text { del MOPT }\end{array}$ & Heredia & M. Herrera & $20 / 01 / 2009$ \\
\hline & $\begin{array}{l}\text { Del Mall Internacional } 300 \text { metros hacia el } \\
\text { aeropuerto (Phillips) } 20 \text { metros aguas abajo del } \\
\text { puente peatonal }\end{array}$ & Alajuela & M. Herrera & $19 / 05 / 2009$ \\
\hline & $\begin{array}{l}\text { Moravia. San Blas de Moravia. Puente en curva } \\
\text { entre San Blas y San Antonio de Coronado }\end{array}$ & San José & M. Herrera & $19 / 03 / 2009$ \\
\hline & $\begin{array}{l}\text { Concepción de la Unión. Cartago. Del Templo } \\
\text { Católico } 200 \text { metros al oeste en puente de calle } \\
\text { a San José }\end{array}$ & Cartago & M. Herrera & 03/03/2009 \\
\hline & $\begin{array}{l}\text { La Guácima. Calle entre la Guácima y Rincón } \\
\text { de Herrera }\end{array}$ & Alajuela & M. Herrera & $06 / 10 / 2008$ \\
\hline
\end{tabular}


Cuadro 1. Continúa

\begin{tabular}{|c|c|c|c|c|}
\hline Taxon & Sitio & Localidad & Recolector & Fecha \\
\hline \multirow{8}{*}{ Erpobdella triannulata } & $\begin{array}{l}\text { Guadalupe, Goicoechea. Calle entre La Paulina } \\
\text { y Guadalupe }\end{array}$ & San José & M. Herrera & $31 / 03 / 2009$ \\
\hline & $\begin{array}{l}\text { Purral de Goicoechea. Cruce a Guadalupe, } \\
\text { Purral y Coronado }\end{array}$ & San José & M. Herrera & $17 / 04 / 2009$ \\
\hline & Curridabat. Puente en Calle hacia San Pedro & San José & M. Herrera & 03/04/2009 \\
\hline & $\begin{array}{l}\text { Sabana Sur, punto en puente de Bajo Los } \\
\text { Anonos, entrada por calle al frente de Embajada } \\
\text { de Brasil }\end{array}$ & San José & M. Herrera & $28 / 04 / 2009$ \\
\hline & $\begin{array}{l}\text { La Guácima. Calle entre la Guácima y Rincón } \\
\text { de Herrera }\end{array}$ & Alajuela & M. Herrera & $19 / 02 / 2009$ \\
\hline & Terminal de Buses de Limón (caribeños) & San José & M. Herrera & $31 / 03 / 2009$ \\
\hline & $\begin{array}{l}\text { Ventanas, Turrúcares, calle a Piedras Negras de } \\
\text { Mora, calle de lastre }\end{array}$ & Alajuela & M. Herrera & $13 / 01 / 2009$ \\
\hline & Parque Nacional Palo Verde & Guanacaste & J. Montemarano & 07/07/2010 \\
\hline \multirow[t]{2}{*}{ Semiscolex sp. } & Parque Nacional Palo Verde & Guanacaste & J. Montemarano & 07/07/2010 \\
\hline & Parque Nacional Palo Verde & Guanacaste & J. Montemarano & $\mathrm{ND} / 07 / 2010$ \\
\hline \multicolumn{5}{|l|}{ Rhynchobdellida } \\
\hline \multirow[t]{2}{*}{ Haementeria sp. } & Finca La Selva & Heredia & D. C. Robinson & 06/11/1966 \\
\hline & Humedal Viejo & Guanacaste & J. Montemarano & $30 / 06 / 2010$ \\
\hline \multirow[t]{3}{*}{ Helobdella cf. stagnalis } & Quebrada Norberta & Cartago & $\begin{array}{l}\text { B. Pacheco y M. } \\
\text { Masis }\end{array}$ & $21 / 04 / 2008$ \\
\hline & Limonal, Esparza & Puntarenas & $\begin{array}{l}\text { P. Gutiérrez y A. } \\
\text { Castillo }\end{array}$ & $25 / 06 / 2008$ \\
\hline & Laguna Peje, 30msnm & Limón & $\begin{array}{l}\text { B. Pacheco y F. } \\
\text { Reyes }\end{array}$ & $31 / 01 / 2012$ \\
\hline \multirow[t]{10}{*}{ Helobdella $\mathrm{cf}$. triserialis } & $\begin{array}{l}\text { La Guácima. Calle entre la Guácima y Rincón } \\
\text { de Herrera }\end{array}$ & Alajuela & M. Herrera & $19 / 02 / 2009$ \\
\hline & $\begin{array}{l}\text { La Guácima. Calle entre la Guácima y Rincón } \\
\text { de Herrera }\end{array}$ & Alajuela & M. Herrera & $19 / 02 / 2009$ \\
\hline & Quebrada Norberta & Cartago & $\begin{array}{l}\text { B. Pacheco\&M. } \\
\text { Masis }\end{array}$ & $21 / 04 / 2008$ \\
\hline & $\begin{array}{l}\text { Moravia. San Blas de Moravia. Puente en curva } \\
\text { entre San Blas y San Antonio de Coronado }\end{array}$ & San José & M. Herrera & $19 / 03 / 2009$ \\
\hline & $\begin{array}{l}\text { La Guácima. Calle entre la Guácima y Rincón } \\
\text { de Herrera Moravia }\end{array}$ & Alajuela & M. Herrera & $06 / 10 / 2008$ \\
\hline & $\begin{array}{l}\text { Guadalupe, Goicoechea. Calle entre La Paulina } \\
\text { y Guadalupe }\end{array}$ & San José & M. Herrera & $31 / 03 / 2009$ \\
\hline & $\begin{array}{l}\text { Purral de Goicoechea. Cruce a Guadalupe, } \\
\text { Purral y Coronado }\end{array}$ & San José & M. Herrera & $17 / 04 / 2009$ \\
\hline & $\begin{array}{l}\text { Rotonda Juan Pablo II y Plaza Mayor de } \\
\text { Rohmoser }\end{array}$ & San José & M. Herrera & $14 / 04 / 2009$ \\
\hline & Parque Nacional Palo Verde & Guanacaste & J. Montemarano & 07/07/2010 \\
\hline & Cuenca del Río Purires & Cartago & L. M. Rodríguez & ND/ND/2010 \\
\hline Helobdella elongata & La Guácima. Calle entre la Guácima y Rincón & Alajuela & M. Herrera & $19 / 02 / 2009$ \\
\hline
\end{tabular}


Cuadro 1. Continúa

\begin{tabular}{|c|c|c|c|c|}
\hline Taxon & Sitio & Localidad & Recolector & Fecha \\
\hline & Quebrada Norberta & Cartago & $\begin{array}{l}\text { B. Pacheco \& M. } \\
\text { Masis }\end{array}$ & $21 / 04 / 2008$ \\
\hline \multirow{9}{*}{ Helobdella sp. } & Embalse Angostura, Turrialba & Cartago & M. Herrera & $10 / 07 / 2009$ \\
\hline & Terminal de Buses de Limón (caribeños) & San José & M. Herrera & $31 / 03 / 2009$ \\
\hline & Peñas Blancas, San Ramón & Alajuela & ND & $14 / 07 / 2003$ \\
\hline & Tierra Blanca & Cartago & R. Araya & $\mathrm{ND} / 05 / 2010$ \\
\hline & Quebrada Mr.Asch, Aguas Zarcas, San Carlos & Alajuela & B. Pacheco & $\mathrm{ND} / \mathrm{ND} / 2009$ \\
\hline & Quebrada Camaroncillo & Limón & X. González & $11 / 03 / 2009$ \\
\hline & $\begin{array}{l}\text { Quebrada Banegas, Pavón, Riyito, R. Rincón, } \\
\text { P. de Osa }\end{array}$ & Puntarenas & ND & $\begin{array}{l}\mathrm{ND} / 10 / 2005 /- \\
\mathrm{ND} / 03 / 2006\end{array}$ \\
\hline & Río Toyogres & Cartago & P. Gutiérrez & ND/02/2009 \\
\hline & Curridabat. Puente en Calle hacia San Pedro & San José & M. Herrera & $27 / 10 / 2008$ \\
\hline \multirow[t]{2}{*}{ Placobdella ringuleti } & $\begin{array}{l}\text { Adherida a una tortuga Kinosternon scorpioides } \\
\text { Ceibo, Cuenca del Río Térraba, Osa }\end{array}$ & Puntarenas & ND & 07/09/2000 \\
\hline & $\begin{array}{l}\text { Adherida a una tortuga Rhinoclemmys annulata, } \\
\text { camino de lastre a Puerto Ecológico Caño } \\
\text { Blanco }\end{array}$ & Limón & B. Pacheco & $17 / 06 / 2010$ \\
\hline Branchellion lobata & Playa Langosta, Santa Cruz & Guanacaste & $\begin{array}{l}\text { Gustavo Serrano } \\
\text { Mora }\end{array}$ & 24/03/1992 \\
\hline Stibarobdella macrothela & Reserva Absoluta, Cabo Blanco & Puntarenas & Luis Mena. & 09/02/1993 \\
\hline
\end{tabular}

por Raúl Ringuelet en 1982 quien los determinó como Limnobdella sp. Sin embargo, dichos ejemplares fueron identificados por nosotros como Cylicobdella costaricae. Otro ejemplar, el CR 07-01 (sin datos de recoleta) fue determinado como Blanchardiella sp. por Ringuelet, de acuerdo a nuestras observaciones corresponden también con C. costaricae.

\section{Comentarios taxonómicos}

La descripción original de Cylicobdella costaricae elaborada por Plotnikow (1905), así como la breve nota de Ringuelet (1981b) constituyen los únicos registros de campo de esta especie. La mención de este taxón en Ringuelet (1944) y Sawyer (1986) solo hacen referencia a los trabajos previamente señalados y no a observaciones nuevas. La descripción original, al igual que la mayoría de los trabajos de la época, se basa únicamente en caracteres ectosomáticos. Dicha descripción menciona que los ejemplares fueron recolectados en Costa Rica, sin especificar el sitio. Los ejemplares estudiados por nosotros provienen de la provincia de San José y de Guanacaste; todos se reconocen como un único taxón, cuyos caracteres morfológicos son compatibles con los señalados por Plotnikow (1905), por lo cual establecemos que los ejemplares aquí estudiados corresponden a C. costaricae.
Sawyer (1986) reconoció además de C. costaricae, otras tres especies más para el género: Cylicobdella joseensis (Grube y Örsted, 1859), Cylicobdella coccinea Kennel, 1886 y Cylicobdella intermedia (Nonato, 1946). El número de anillos separando los gonoporos es el carácter diagnóstico de cada una de las especies. En el caso de C. costaricae hay $1+1 / 2$ anillos separando los gonoporos, mientras que en $C$. coccinea hay $1 / 2+1+1 / 2$ o bien $2+1 / 2$, mientras que en $C$. joseensis y $C$. intermedia, hay 2 anillos completos separando los gonoporos.

Estrechamente relacionadas con las especies del género Cylicobdella se encuentran las pertenecientes a los géneros Blanchardiella y Lumbricobdella. La distinción entre cada uno de estos géneros no ha sido estudiada a fondo y dada la gran similitud ecológica y morfológica de las especies de estos géneros es posible que en realidad, pueda tratarse de un solo grupo. Las especies de los 3 géneros comparten la forma general del cuerpo, la forma del aparato digestivo desde la boca hasta el ano, la disposición del aparato reproductor masculino y femenino, además de la distribución geográfica. Se ha señalado que las especies de Blanchardiella, a diferencia de las de Cylicobdella, presentan mandíbulas rudimentarias con estiletes además de manchas oculares, estas últimas supuestamente ausentes en la especies del género Cylicobdella, pero presentes en C. costaricae, como se señala con anterioridad. Considera- 
mos que conceder importancia taxonómica a las manchas oculares es problemático, ya que en muchos organismos, los pigmentos desaparecen aparentando la ausencia de dicho carácter. Por otra parte, las especies de Lumbricobdella presentan 10 o más anillos y múltiples testisacos por somito, contrastando con el somito 5 anillado y con el único par de testisacos por somito característico de las especies de Cylicobdella y Blanchardiella. El análisis detallado de la morfología de la región anterior, incluyendo la apertura oral y la faringe de los ejemplares aquí estudiados, nos permite afirmar que no presentan mandíbulas rudimentarias, estiletes ni ninguna estructura esclerotizada; sin embargo, la forma en que los pliegues musculares se arreglan en la faringe podrían asemejarse a la forma de las mandíbulas características de otros Hirudiniformes (e.g. Hirudo medicinalis Linnaeus, 1758), sin embargo es claro que en $C$. costaricae dichas estructuras están ausentes.

La disección de los ejemplares de Cylicobdella costaricae empleados para la redescripción que se presenta aquí nos permitió examinar el contenido del aparato digestivo. En el buche de un ejemplar se hallaron restos de oligoquetos así como también individuos pequeños de $C$. costaricae, confirmándose que el canibalismo está presente en esta especie, al igual que en otras especies macrófagas (Davies y Everett, 1975).

\section{Otros registros}

Orden Arhynchobdellida Blanchard, 1894

Familia Salifidae Johanson, 1910

Género Barbronia Johanson, 1918

Barbronia weberi Blanchard, 1897 (Figs. 4, 5).

Cuerpo largo y con ancho uniforme, no aplanados dorsoventralmente, clitelo evidente en ejemplares adultos, ventosas poco desarrolladas. Color pardo uniforme, ligeramente más claro en la superficie ventral, con 3 pares de manchas oculares, un par anterior y 2 pares posteriores. Boca amplia en el centro de la ventosa anterior. Un par de gastroporos en la línea media ventral, el primero anterior al gonoporo masculino y el segundo posterior al gonoporo femenino. Organismos de agua dulce, macrófagos (Sawyer, 1986). Especie considerada invasora, originaria del sureste asiático o África, que ha sido introducida accidentalmente alrededor del mundo (Genoni y Fazzone, 2008; OcegueraFigueroa et al., 2011).

Orden Arhynchobdellida Blanchard, 1894

Familia Erpobdellidae Blanchard, 1894

Género Erpobdellade Blainville, 1818

Erpobdella triannulata Moore 1908 (Fig. 6).

Cuerpo largo y con ancho uniforme, no aplanado dorsoventralmente, clitelo evidente en ejemplares adul- tos, ventosas poco desarrolladas. Color pardo o gris claro, algunos ejemplares tienen franjas longitudinales de posición dorsal. Tres pares de manchas oculares, un par anterior y 2 pares posteriores. Boca amplia en el centro de la ventosa anterior. Gonoporos separados por 3 anillos. Sin gastroporos. Organismos de agua dulce, macrófagos (Sawyer, 1986).

Orden Arhynchobdellida Blanchard, 1894

Familia Semiscolecidae Ringuelet, 1972

Género Semiscolex Kinberg, 1866

Semiscolex sp. (Fig. 7).

Cuerpo largo y muscular con ancho uniforme, el extremo anterior ligeramente adelgazado. No aplanados dorsoventralmente, clitelo evidente, ventosas poco desarrolladas. Color pardo con un par de franjas paramedianas longitudinales de color pardo obscuro. Cinco pares de manchas oculares arregladas en arco. Boca amplia en el centro de la ventosa anterior. Gonoporos separados por 13 1/2 anillos. Sin gastroporos. Las características morfológicas de estos organismos no corresponden con las descritas para ninguna especie del género (ver Ringuelet, 1985) y probablemente se trata de una especie aún no descrita. Organismos de agua dulce, macrófagos (Sawyer, 1986).

Orden Rhynchobdellida Blanchard, 1894

Familia Glossiphoniidae Vaillant, 1890

Género Haementeria de Filippi, 1849

Haementeria sp. (Fig. 8).

Se examinaron dos ejemplares en malas condiciones de conservación. Cuerpo aplanado dorsoventralmente, lanceolado, clitelo no evidente, ventosa anterior poco desarrollada con la boca en forma de poro situada en el margen anterior, ventosa posterior fuerte y fácilmente distinguible. Tegumentos blanquecinos y ligeramente transparentes. Un único par de manchas oculares muy próximos entre sí. Superficie dorsal con 11 hileras longitudinales de papilas. Las especies de Haementeria son dulceacuícolas, estrictamente hematófagasy se alimentan de una gran variedad de especies de vertebrados (Oceguera-Figueroa, 2011).

Orden Rhynchobdellida Blanchard, 1894

Familia Glossiphoniidae Vaillant, 1890

Género Helobdella Blanchard, 1896

Helobdella sp. (Fig. 9).

Cuerpo aplanado dorsoventralmente, región anterior estrecha que se ensancha hacia el extremo posterior, lanceolado; clitelo no evidente, ventosa anterior poco desarrollada con la boca en forma de poro situada en el margen anterior, ventosa posterior fuerte y fácilmente distinguible. Tegumentos blanquecinos. Un par de manchas oculares bien separadas entre sí. Sin placa quitinoide 

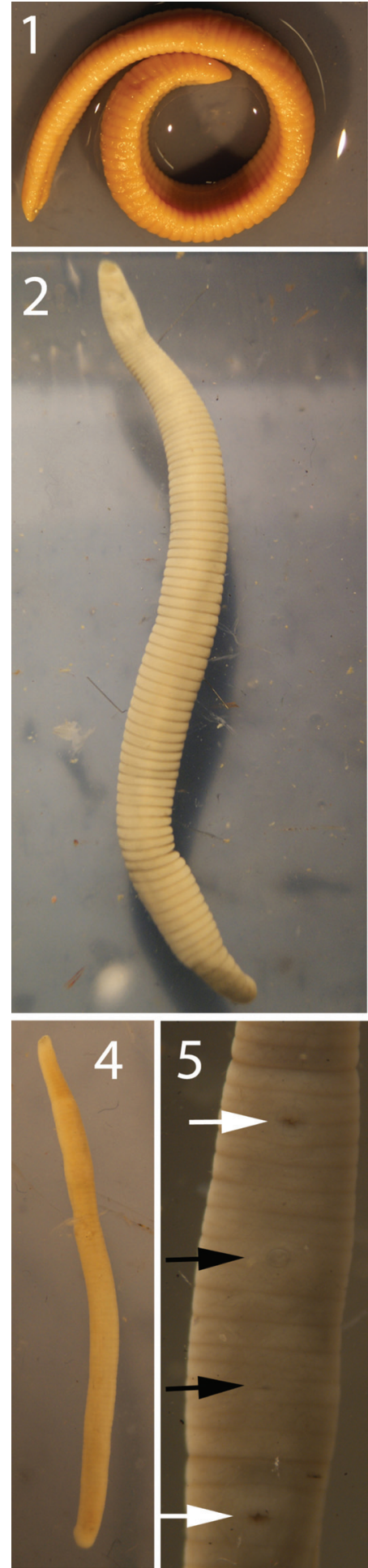

3

XII

XIII

XIV

XV

XVI
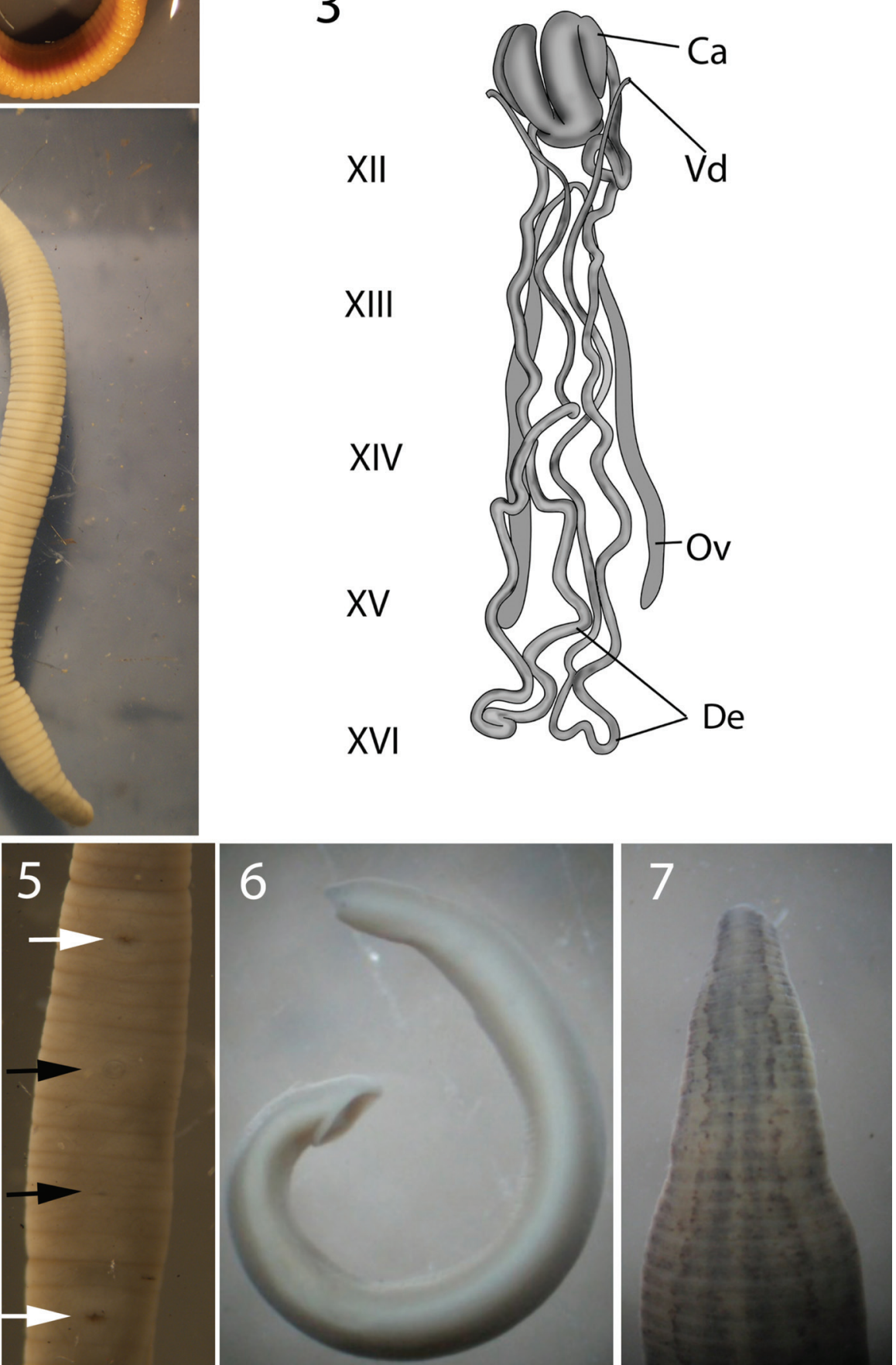

Figuras 1-7. Arhynchobdellida de Costa Rica. 1. Vista lateral de Cylicobdella costaricae. 2. Vista dorsal de C. costaricae. 3. Vista dorsal del aparato reproductor masculino y femenino de C. costaricae. (Ca) Cuernos atriales; (Vd) Vaso deferente; (De) Ducto eyaculatorio; (Ov) Ovario. 4. Vista ventral de Barbronia weberi. 5. Detalle de la vista ventral de los somitos sexuales de $B$. weberi. Flechas blancas señalan los gastroporos y flechas negras señalan el gonoporo masculino y femenino. 6. Vista lateral de Erpobdella triannulata.7. Vista dorsal del extremo anterior de Semiscolex sp. 
dorsal. Sin papilas, sensilas o manchas arregladas metamericamente. Las características morfológicas de estos ejemplares no corresponden con las de las especies descritas con anterioridad para Costa Rica y posiblemente se trate de una especie nueva. Sin embargo, la taxonomía del grupo requiere de una revisión formal ya que se ha demostrado que existen diversas formas únicamente distinguibles con base en información molecular (Oceguera-Figueroa et al., 2010). Las especies del grupo se consideran dulceacuícolas y liquidosomatófagas (Sawyer, 1986).

Orden Rhynchobdellida Blanchard, 1894

Familia Glossiphoniidae Vaillant, 1890

Género Helobdella Blanchard, 1896

Helobdella cf. stagnalis (Linnaeus, 1758) (Figs. 10, 11).

Cuerpo aplanado dorsoventralmente, región anterior estrecha y se ensancha hacia el extremo posterior, lanceolado, clitelo no evidente, ventosa anterior poco desarrollada con la boca en forma de poro situada en el margen anterior, ventosa posterior fuerte y fácilmente distinguible. Tegumentos blanquecinos. Un par de manchas oculares bien separadas entre sí. Placa quitinoide dorsal de forma triangular en el extremo anterior del cuerpo. Un ejemplar fue colectado sobre una tortuga Kinosternon scorpioides (Linnaeus, 1766), sin embargo, las especies de Helobdella no se alimentan de sangre de vertebrados por lo que se considera que su presencia en la tortuga es accidental. La taxonomía del grupo requiere de una revisión formal ya que se ha demostrado que existen diversas formas únicamente distinguibles en base a información del ADN (Oceguera-Figueroa et al., 2010). Las especies del grupo se consideran dulceacuícolas y liquidosomatófagas (Sawyer, 1986).

Orden Rhynchobdellida Blanchard, 1894

Familia Glossiphoniidae Vaillant, 1890

Género Helobdella Blanchard, 1896

Helobdella $\mathrm{cf}$.triserialis (E. Blanchard, 1849) (Figs. 12, 13).

Cuerpo aplanado dorso-ventralmente, lanceolado, con la región anterior estrecha y ensanchándose hacia el extremo posterior; clitelo no evidente, ventosa anterior poco desarrollada con la boca en forma de poro situada en el margen anterior; ventosa posterior fuerte y fácilmente distinguible. Tegumentos blanquecinos. Un par de manchas oculares bien separadas entre sí. Sin placa quitinoide dorsal, 3 o más hileras longitudinales dorsales de papilas y pigmentos muy evidentes. Las especies del grupo se consideran dulceacuícolas y liquidosomatófagas (Sawyer, 1986).

Orden Rhynchobdellida Blanchard, 1894

Familia Glossiphoniidae Vaillant, 1890

Género Helobdella Blanchard, 1896
Helobdella elongata (Castle, 1900) (Fig. 14).

Cuerpo no aplanado dorso-ventralmente, ancho uniforme a lo largo del cuerpo, circular o semicircular en corte transversal, ventosas poco desarrolladas. Boca en forma de poro situada en el margen anterior de la ventosa oral. Clitelo no evidente. Tegumentos blanquecinos. Un par de manchas oculares bien separadas entre sí. Sin placa quitinoide dorsal, sin pigmentos o papilas arregladas metaméricamente. Las especies del grupo se consideran dulceacuícolas y liquidosomatófagas (Sawyer, 1986).

Orden Rhynchobdellida Blanchard, 1894

Familia Glossiphoniidae Vaillant, 1890

Género Placobdella Blanchard, 1893

Placobdella ringueleti López-Jiménez y Oceguera-Figueroa, 2009 (Figs. 15, 16).

Ejemplares recolectados parasitando tortugas de agua dulce Kinosternon scorpioides y Rhinoclemmys annulata (Gray, 1860). Cuerpo aplanado dorso-ventralmente, parte anterior estrecha y se ensancha hacia el extremo posterior, lanceolado, clitelo no evidente, ventosa anterior poco desarrollada con la boca en forma de poro, situada en el margen anterior, ventosa posterior fuerte y fácilmente distinguible. Cuerpo blanquecino y de tegumento ligeramente transparente. Dos pares de manchas oculares, el primer par en coalescencia, el segundo par ligeramente posterior al primero, dando la apariencia de formar una sola mancha ocular. Una única línea longitudinal dorsal de color pardo, en algunos ejemplares, dicha línea se encuentra desvanecida. Los caracteres morfológicos se corresponden a los descritos por López-Jiménez y Oceguera-Figueroa (2009). Las especies de Placobdella son estrictamente de agua dulce y hematófagas, particularmente de tortugas de agua dulce (Oceguera-Figueroa y Siddall, 2008; López-Jiménez y Oceguera-Figueroa, 2009).

Orden Rhynchobdellida Blanchard, 1894

Familia Piscicolidae Johnston, 1865

Género Branchellion Savigny, 1822

Branchellion lobata Moore, 1952 (Fig. 17).

Colectados de la cloaca de una raya hembra. Cuerpo semicircular en corte transversal, no aplanados dorsoventralmente, ancho del cuerpo más o menos uniforme distinguiéndose claramente 2 regiones, una anterior (traquelosoma) y otra posterior (urosoma). El traquelosoma está provisto de 31 pares de branquias laterales en forma de lámina, clitelo no distinguible. Un par de manchas oculares, ventosas bien desarrolladas en forma de copa. Los caracteres morfológicos son consistentes a los descritos por Sawyer (1986) y Moore (1946). Las especies del grupo son exclusivamente marinas y hematófagas, generalmente se alimentan de sangre de rayas y tiburones (Sawyer, 1986). 


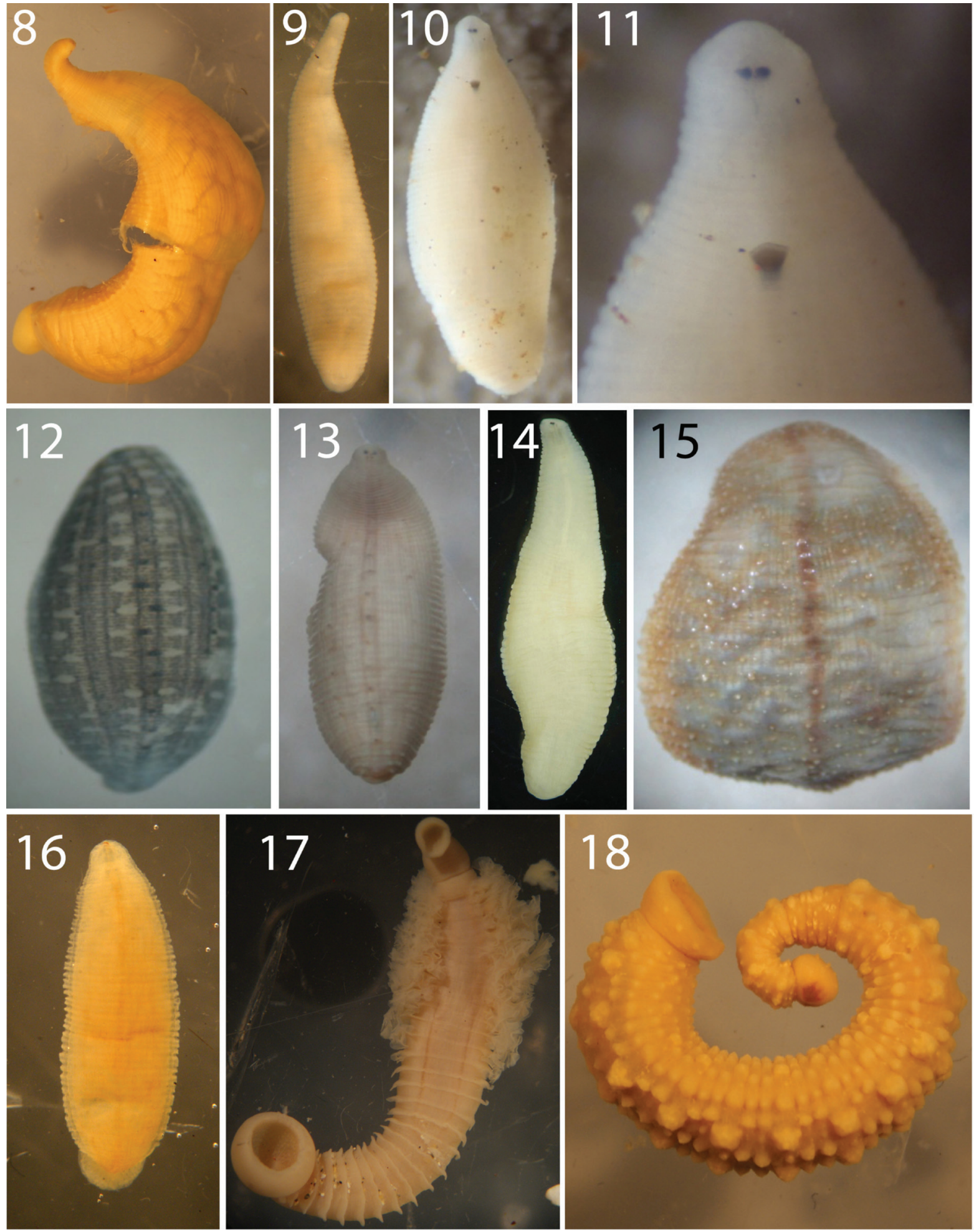

Figuras 8-18. Rhynchobdellida de Costa Rica. 8. Vista lateral de Haementeria sp. 9. Vista dorsal de Helobdella sp. 10 Vista dorsal de Helobdella cf. stagnalis. 11. Detalle de la placa quitinoide dorsal de Helobdella cf. stagnalis. 12 y 13. Vista dorsal de Helobdella cf. triserialis. 14. Vista dorsal de Helobdella elongata. 15 y 16. Vista dorsal de Placobdella ringueleti. 17. Vista ventral de Branchellion lobata. 18. Vista lateral de Stibarobdella macrothela. 
Orden Rhynchobdellida Blanchard, 1894

Familia Piscicolidae Johnston, 1865

Género Stibarobdella Leigh-Sharpe, 1925

Stibarobdella macrothela (Schmarda, 1861) (Fig. 18).

Un ejemplar. Cuerpo semicircular en corte transversal, clitelo no distinguible. Ancho del cuerpo parcialmente uniforme, más ancho hacia la mitad del cuerpo y el extremo anterior es más estrecho. Ventosas bien desarrolladas en forma de copa. Superficie del cuerpo cubierta completamente por papilas prominentes. Color blanquecino. Las especies del grupo se consideran exclusivamente marinas y hematófagas, generalmente se alimentan de sangre de rayas y tiburones (Sawyer, 1986).

\section{Discusión}

El conocimiento de las sanguijuelas de diversas regiones del continente americano es aún escaso, con pocos registros publicados de forma aislada. Esto es particularmente cierto para los países centroamericanos, por lo cual el presente trabajo representa el punto de partida para estudios futuros, no sólo de Costa Rica sino de los países adyacentes. Este estudio representa el primer trabajo que específicamente investiga la diversidad de sanguijuelas de Costa Rica y se reportan 63 registros pertenecientes a 12 especies recolectadas en las 7 provincias que conforman la República de Costa Rica.

De las 12 especies registradas, 4 pertenecen al orden Arhynchobdellida: Cylicobdella costaricae, Erpobdella triannulata, Barbronia weberi y Semiscolex sp. y 8 taxones pertenecen al orden Rhynchobdellida: Haementeria sp., Helobdella sp. Helobdella cf. stagnalis, Helobdella cf. triserialis, Helobdella elongata, Placobdella ringueleti, Branchellion lobata y Stibarobdella macrothela. Dos de ellas: Branchellion lobata y Stibarobdella macrothela, son exclusivamente marinas y parásitas de elasmobranquios. El resto de las especies, con excepción de Cylicobdella costaricae que es terrestre, habitan ambientes dulceacuícolas, tanto ríos como lagunas.

En cuanto a la distribución geográfica de los hirudíneos en Costa Rica, en las provincias de Cartago y Guanacaste se registró el mayor número de especies (6), seguidas por San José y Alajuela con 5 especies cada una, Puntarenas con 4 y finalmente Heredia y Limón con 3 especies cada una. En cuanto al número de registros de hirudíneos, San José es la provincia con mayor número (24) y Limón en el otro extremo con solo 3 registros. Por otra parte, es interesante destacar que la especie invasora, Barbronia weberi se encuentra ampliamente distribuida en el país, con registros en 5 de las 7 provincias que lo constituyen (San José, Cartago, Alajuela, Heredia y Guanacaste). El impacto de esta especie en las comunidades animales de agua dulce no ha sido aún evaluado, sin embargo al tratarse de un organismo depredador, macrófago y muy voraz, existe la posibilidad de que se afecte a las poblaciones de invertebrados e inclusive que se alimente de huevos de anfibios y peces de diversas especies.

En Costa Rica confluyen una gran diversidad de organismos con afinidades biogeográficas tanto con el Neártico como con el Neotrópico (ver Savage, 2002) y el caso de los hirudíneos no es distinto. Las especies Erpobdella triannulata y Placobdella ringueleti pertenecen claramente a linajes neárticos (Siddall et al., 2005; Oceguera-Figueroa et al., 2010, 2012), sin embargo el resto de la fauna está mayoritariamente conformada por elementos neotropicales, como las diversas especies de Helobdella, Haementeria sp. Semiscolex sp. y Cylicobdella (Ringuelet, 1985; Siddall et al., 2002; Oceguera-Figueroa 2011). Este ensamblaje de especies con afinidades hacia las 2 grandes regiones biogeográficas, hacen de Costa Rica un lugar de gran importancia para el estudio de la biodiversidad. El ensamblaje de comunidades y la conservación son áreas en las cuales el conocimiento de los hirudíneos podría aportar información clave hasta ahora no considerada.

Clave taxonómica para determinar las especies de sanguijuelas (Annelida: Clitellata) de Costa Rica.

1. Ventosa anterior y posterior bien desarrolladas y claramente distinguibles, en forma de copa. Cuerpo semicircular en corte transversal, organismos marinos.

1.1 Ventosa anterior poco desarrollada, ventosa posterior bien desarrollada en algunas especies. Organismos dulceacuícolas y terrestres. Branchellion lobata

2. Cuerpo con branquias laterales. Stibarobdella macrothela

2.1 Cuerpo sin branquias laterales.

3. Boca en el margen anterior de la ventosa oral, en forma de un poro pequeño. Con proboscis eversible.

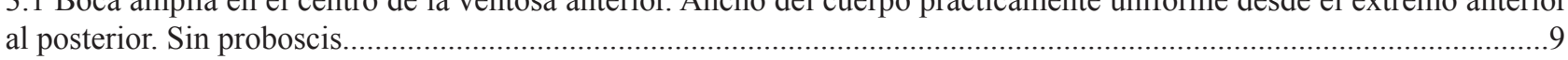

4. Placa quitinoide dorsal en el primer tercio del cuerpo.................................................................. Helobdella cf. stagnalis

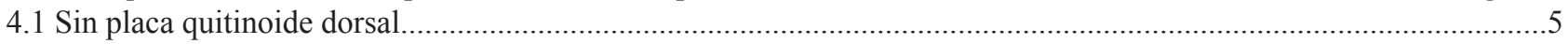

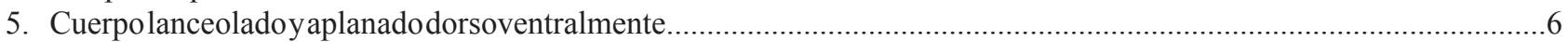


5.1 Cuerpo circular o semicircular en corte transversal. Un par de manchas oculares bien separadas entre sí

6. Sin líneas dorsales, cuerpo de color blanco uniforme......

Helobdella elongata

6.1 Una o más líneas longitudinales en la cara dorsal del cuerpo.

Helobdella $\mathrm{sp}$.

7. Una única línea longitudinal dorsal de color pardo de posición media. Dos pares de manchas oculares, el primer par en coalescencia, el segundo par ligeramente posterior al primer par, dando la apariencia de una sola mancha ocular.

Placobdella ringueleti

7.1 Múltiples líneas longitudinales de posición dorsal, un par de manchas oculares.

Helobdella cf. triserialis

8. Par de manchas oculares bien separadas entres sí. Haementeria sp.

8.1 Par de manchas oculares en coalescencia

Barbronia weberi

9.1 Sin gastroporos.

10. Manchas oculares arregladas en forma de arco.

10.1 Un par de manchas oculares anterior y dos pares posteriores en el mismo anillo, sin gastroporos, tres anillos separando los gonoporos. Erpobdella triannulata

11. Tres o más pares de manchas oculares arregladas en forma de arco Cylicobdella costaricae 11.1 Cinco pares de manchas oculares arregladas en arcos, $13 \frac{1}{2}$ anillos entre los gonoporos Semiscolex sp.

\section{Agradecimientos}

A la Dirección General de Asuntos del Personal Académico de la UNAM y al CONACYT (Propuesta 165414) por las becas otorgadas al primer autor. Anna Phillips colaboró en la elaboración del resumen en inglés. Monika Springer y Rita Vargas del Museo de Zoología de la Universidad de Costa Rica facilitaron el acceso al material depositado previamente. Mauricio Herrera y Silvia Echeverría de la Universidad Nacional de Costa Rica donaron especímenes de sus colecciones.

\section{Literatura citada}

Blanchard, E. 1849. Annelides. In Historia Física y Política de Chile, Zoología, D. C. Gay (ed.). Paris 3. p. 43-50.

Caballero y C., E. 1941. Hirudíneos de México XVI. Nuevos huéspedes y localidades para algunas sanguijuelas ya conocidas y descripción de una nueva especie. Anales del Instituto de Biología. Universidad Nacional Autónoma de México, Serie Zoología 12:747-757.

Caballero y C., E. 1956. Hirudineos de México XX. Taxa y nomenclatura de la clase Hirudinea hasta géneros. Anales del Instituto de Biología. Universidad Nacional Autónoma de México, Serie Zoología 27:279-302.

Caballero y C., E. 1959. Hirudíneos de México XXII. Taxa y nomenclaturade la clase Hirudinea hasta géneros (Nueva edición). Anales del Instituto de Biología. Universidad Nacional Autónoma de México, Serie Zoología 30:227-242.

Cordero, E. H. 1937. Hirudíneos neotropicales y subantárticos. Nuevos, críticos o ya conocidos del Museo Argentino de Ciencias Naturales. Anales del Museo Argentino de Ciencias Naturales 39:1-78.

Davies, R. W. y R. P. Everett. 1975. The feeding of four species of freshwater Hirudinoidea in Southern Alberta. Verhandlungen des Internationalen Verein Limnologie 19:2816-2827.

Davies, R. W. y F. R. Govedich. 2001. Annelida: Euhirudinea and Acanthobdellidae. In Ecology and Classification of North American Freshwater Invertebrates. Second Edition. J. H. Thorp y A.P. Covich (eds.) Academic Press, San Diego. p. 465-504.

Dequal, L. 1917. Nuovi Irudinei esotici del Museo Zoologico di Torino. Bollettino del Museo regionale di scienze naturali di Torino 32:1-20.

Genoni, P. y A. Fazzone. 2008. Barbronia weberi (R. Blanchard, 1897) (Hirudinea: Salifidae), an Asian leech species new to Italy. Aquatic Invasions 3:77-79.

Klemm, D.J. 1982. The leeches (Annelida: Hirudinea) of North America. Cincinnati: Aquatic Biology Section, Environmental Monitoring and Support Laboratory. Office of Research and Development, U.S. Environmental Protection Agency.

López-Jiménez, S. y A. Oceguera-Figueroa. 2009. New Species of Rhynchobdellid Leech (Hirudinea: Glossiphoniidae), a parasite of turtles from Chiapas, Mexico. Journal of Parasitology 95:1356-1359.

Madill, J.y P. Hovingh. 2007. Freshwater leech (Annelida: Hirudinida) distribution in the Canadian province of Newfoundland and Labrador and adjacent regions: checklist, new records, new pigmentation forms, and Pleistocene refugia. Zootaxa 1657:1-21.

Moore, J.P. 1946. Leeches (Hirudinea) from the Hawaiian islands, and two new species from the Pacific region in the Bishop Museum collection. Occasional Papers of Bernice P. Bishop Museum 18:171-191.

Moore, J. P. 1953. Three undescribed species north American leeches (Hirudinea). Notulae Naturae of the Academy of 
Natural Sciences of Philadelphia 250:1-30.

Nakano, T., Z. Ramlah y T. Hikida. 2012. Phylogenetic position of gastrostomobdellid leeches (Hirudinida, Arhynchobdellida, Erpobdelliformes) and a new family for the genus Orobdella. Zoologica Scripta 41:177-185.

Oceguera-Figueroa, A. 2011.Molecular phylogeny of the New World bloodfeeding leeches of the genus Haementeria and reconsideration of the biannulate genus Oligobdella. Molecular Phylogenetics and Evolution 62:508-514.

Oceguera-Figueroa, A., A. K. Barrio, M. I. Aldea-Guevara y M. E. Siddall. 2012. Evaluation of the evolution of jaw morphology in New World hirudiniform leeches, with a description of a new blood-feeding species of Oxyptychus (Annelida: Hirudiniformes) from the Peruvian Amazon. Invertebrate Systematics 2012:17-24

Oceguera-Figueroa A., V. León-Règagnon y M. Siddall. 2010. DNA barcoding reveals Mexican diversity within the freshwater leech genus Helobdella (Annelida: Glossiphoniidae). Mitochondrial DNA 21:24-29.

Oceguera-Figueroa, A., A. J. Phillips, B. Pacheco-Chaves, W. K. Reeves y M. E. Siddall. 2010. Phylogeny of macrophagous leeches (Hirudinea, Clitellata) based on molecular data and evaluation of the barcoding locus. Zoologica Scripta 40:194-203.

Oceguera-Figueroa, A. y M. E. Siddall. 2008. Placobdella lamothei n. sp. (Hirudinea: Glossiphoniidae), a new leech parasite of freshwater turtles from Estado de México, Mexico. Revista Mexicana de Biodiversidad 79:135-139.

Pacheco-Chaves, B. 2010. Diversidad taxonómica y distribución de chinches patinadores (Hemiptera: Gerridae) en Costa Rica. Monografía, Escuela de Biología, Facultad de Ciencias, Universidad de Costa Rica. San José, Costa Rica. 128 p.

Plotnikow, V. 1905. Glossosiphoniidae, Hirudinidae et Herpobdellidae Zoologiceskago Museia Imperatorkoia Akademii. Annuaire du Musée Zoologique de l'Académie Imperiale des Sciences. St. Petersburg. Akademiya Nauk,
Rusia 10:133-158.

Ringuelet, R. A. 1944. Revisión de los hirudíneos argentinos de los géneros Helobdella R. B1. Batracobdella Vig., Cylicobdella Gr. y Semiscolex Kinb. Revista del Museo de la Plata (Nueva Serie). Sección Zoología 4:5-94.

Ringuelet, R. A. 1981a. Clave para el reconocimiento de los hirudíneos de México. Anales del Instituto de Biología, Universidad Nacional Autónoma de México, Serie Zoología 52:89-97.

Ringuelet, R. A. 1981b. Some advances in the knowledge of Neotropical Leeches. Limnobios 2:2-3.

Ringuelet, R. A. 1982. Hirudinea. In Aquatic biota of Mexico, Central America and the West Indies, S. H. Hurlbert y A. Villalobos-Figueroa (eds.) San Diego State University Press. San Diego. p. 166-171.

Ringuelet, R. A. 1985. Fauna de Agua dulce de la República Argentina. Hirudinea, FECIC, Buenos Aires, 321 p.

Savage, J. M. 2002. The Amphibians and Reptiles of Costa Rica: A Herpetofauna between two Continents, between Two Seas. University of Chicago Press, Chicago, 934 p.

Sawyer, R.T. 1967.The leeches of Louisiana, with notes on some North American species (Hirudinea, Annelida). Proceedings of the Louisiana Academy of Science 30:32-38.

Sawyer, R. T. 1986. Leech Biology and Behaviour. Clarendon Press, Oxford. 1065 p.

Siddall, M. E. y E. Borda. 2002. Phylogeny of the leech genus Helobdella (Glossiphoniidae) based on mitochondrial gene sequences and morphological data and a special consideration of the triserialis complex. Zoologica Scripta 32:23-33.

Siddall, M. E., R. B. Budinoff y E. Borda. 2005. Phylogenetic evaluation of systematics and biogeography of the leech family Glossiphoniidae. Invertebrate Systematics 19:105112.

Springer, M., A. Ramírez y P. Hanson (eds.). 2010. Macroinvertebrados de agua dulce de Costa Rica 1. Revista de Biología Tropical. 58 (Suplemento 4). 240 p. 